\title{
OPEN Biodegradation and metabolic pathway of sulfamethoxazole by Sphingobacterium mizutaii
}

\author{
Jinlong Song ${ }^{1,5}$, Guijie Hao ${ }^{2,5}$, Lu Liu ${ }^{1}$, Hongyu Zhang ${ }^{1}$, Dongxue Zhao ${ }^{1,3}$, Xingyang $\mathrm{Li}^{1,3}$, \\ Zhen Yang ${ }^{1}$, Jinhua Xu ${ }^{1}$, Zhiyong Ruan ${ }^{4} \bowtie$ \&ingchun Mu ${ }^{1 凶}$
}

Sulfamethoxazole (SMX) is the most commonly used antibiotic in worldwide for inhibiting aquatic animal diseases. However, the residues of SMX are difficult to eliminate and may enter the food chain, leading to considerable threats on human health. The bacterial strain Sphingobacterium mizutaii LLE5 was isolated from activated sludge. This strain could utilize SMX as its sole carbon source and degrade it efficiently. Under optimal degradation conditions $\left(30.8^{\circ} \mathrm{C}, \mathrm{pH} 7.2\right.$, and inoculum amount of $3.5 \times 10^{7} \mathrm{cfu} / \mathrm{mL}$ ), S. mizutaii LLE5 could degrade $93.87 \%$ of $50 \mathrm{mg} / \mathrm{L} \mathrm{SMX}$ within 7 days. Four intermediate products from the degradation of SMX were identified and a possible degradation pathway based on these findings was proposed. Furthermore, S. mizutaii LLE5 could also degrade other sulfonamides. This study is the first report on (1) degradation of SMX and other sulfonamides by S. mizutaii, (2) optimization of biodegradation conditions via response surface methodology, and (3) identification of sulfanilamide, 4-aminothiophenol, 5-amino-3-methylisoxazole, and aniline as metabolites in the degradation pathway of SMX in a microorganism. This strain might be useful for the bioremediation of SMX-contaminated environment.

The amount of aquaculture in China ranks first in the world all year round. The huge output mainly depends on high-density aquaculture ${ }^{1}$. This type of aquaculture leads to the risk of outbreaks of aquatic animal diseases. Sulfonamides are synthetic drugs used as anti-microbial, anti-diabetic, diuretic, anticonvulsant, and herbicidal agents. Among which, sulfamethoxazole (SMX) is one of the most prescribed antibiotics worldwide, can kill aquatic pathogens and can effectively inhibit aquatic animal diseases ${ }^{2-4}$. However, SMX cannot be completely utilized and metabolized by aquatic animals because approximately $50 \%$ are excreted to the environment without modification. Consequently, SMX has become a widely distributed pollutant in aquatic and domestic waste waters ${ }^{5}$. Numerous studies have indicated that SMX has adverse ecological effects and may threaten human health after long-term exposure. The World Health Organization has classified SMX as a Category 3 carcinogen in $2017^{6}$. However, SMX has been proven to be difficult to eliminate by conventional water treatment processes ${ }^{7}$. The residues of SMX enter the food chain, leading to considerable threats to human health. Therefore, an efficient and reliable degradation method for removing SMX residue from the water environment must be developed ${ }^{8}$.

SMX removal is mainly by physical, chemical, and microbial methods ${ }^{9}$. The physical and chemical methods mainly include anion exchange, nano-filtration, struvite precipitation, and electrodialysis ${ }^{10}$. However, for technical and economic reasons, physical and chemical techniques may not be feasible ${ }^{11}$. Microbial biodegradation has advantages in terms of its eco-friendliness, low cost, and scope of implementation and has been proven as an effective method for the remediation of SMX residues in aquaculture waters ${ }^{12}$. To date, more than 20 strains of SMX-degrading strains have been isolated from different environments, and all of them exhibit SXM-degrading ability under laboratory conditions. Liang et al. isolated Achromobacter sp. JL9, which can use SMX as the sole nitrogen source for growth. The degradation rate of SMX was $90.4 \%$, and the highest reaction rate constant was $0.0384 \mathrm{~min}^{-113}$. Gao et al. found that Phanerochaete chrysosporium has a strong tolerance to sulfamethoxazole in the concentration range of $10-30 \mathrm{mg} / \mathrm{L}^{14}$. At $10 \mathrm{mg} / \mathrm{L}$, the degradation rate reached $53 \%$ after $24 \mathrm{~h}$ and $74 \%$ after 10 days. Jia et al. reported that the sulfate-reducing bacteria (SRB) sludge system shows considerable ability

\footnotetext{
${ }^{1}$ Key Laboratory of Control of Quality and Safety for Aquatic Products (Ministry of Agriculture and Rural Affairs), Chinese Academy of Fishery Sciences, Beijing 100141, China. ${ }^{2}$ Key Laboratory of Healthy Freshwater Aquaculture, Ministry of Agriculture and Rural Affairs, Key Laboratory of Freshwater Aquaculture Genetic and Breeding of Zhejiang Province, Zhejiang Institute of Freshwater Fisheries, Huzhou 313001, China. ${ }^{3}$ College of Food Science and Engineering, Bohai University, Jinzhou 121013, China. ${ }^{4}$ Institute of Agricultural Resources and Regional Planning, CAAS, Beijing 100081, China. ${ }^{5}$ These authors contributed equally: Jinlong Song and Guijie Hao. ${ }^{\square}$ email: ruanzhiyong@caas.cn; muyc@cafs.ac.cn
} 


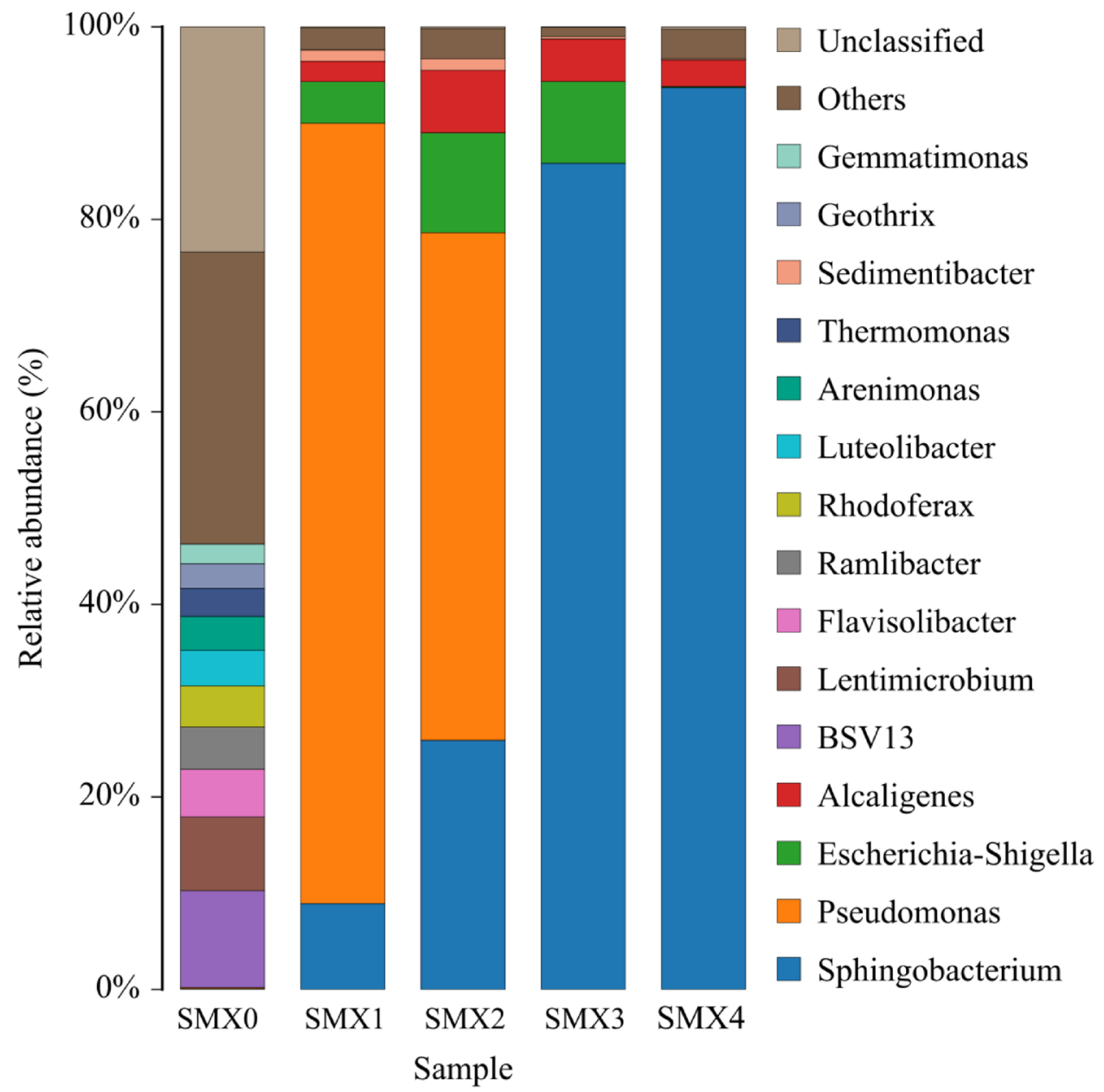

Figure 1. Diversity and community changes in enrichment cultures SMXY, SMX1, SMX2, SMX3 and SMX4 based on the relative abundance of the illumina sequences. The histogram was constructed by using $\mathrm{R}$ software.

to degrade SMX. When the initial concentration is $25,50,100,150$, and $200 \mathrm{mg} / \mathrm{L}$, the removal rates of SMX by SRB sludge through adsorption and biodegradation are 3.9,5.6,13.2, 15.9 , and $21.3 \mathrm{mg} / \mathrm{L} / \mathrm{d}^{15}$. Other bacteria, including Achromobacter denitrificans PR ${ }^{16}$, P. chrysosporium ${ }^{17}$, Pseudomonas stutzeri ${ }^{18}$, and Acinetobacter sp. ${ }^{19}$, could serve as resources for the bioremediation of SMX from the polluted environment. Nevertheless, further studies are needed. Previous studies mainly isolated SMX-degrading bacteria through traditional methods, and the degradation efficiency was relatively low. The degradation mechanism of SMX and the metabolic pathway involved are also unclear.

The aims of this study were: (1) to isolate a bacterial strain that can highly degrade SMX by a novel method; (2) to optimize the environmental parameters to improve degradation efficiency; and (3) to deduce the possible degradation pathway of degrading strain and examine the mechanism underlying SMX degradation.

\section{Results}

Community changes and diversity of SMX-degrading enrichment cultures. A total of 5978, $6012,6048,6005$, and 6003 16S rRNA gene sequences of the distinct V3-V4 regions were obtained from sludge sample (SMXY) and four generations of enrichment cultures (SMX1-4) by high-throughput sequencing, respectively. After statistical analysis and annotation, the results (Fig. 1) showed that 5978 sequences in sludge sample SMXY were clustered into 166 OTUs. The highest abundance of OTUs belonged to bsv13, Lentimicrobium, Flaviolibacter, Ramlibacter, and Rhodoferax. After enrichment, the bacterial diversity of SMX1 rapidly decreased, 6012 sequences were clustered into only 23 OTUs. The main genera were Pseudomonas, Sphingobacterium, Escherichia-Shigella, Alcaligenes, and Sediminibacter. Pseudomonas became the highest abundance genus in SMX2. After the second passage, with the increase in the SMX concentration, the bacterial diversity of SMX2 decreased continuously, and 6048 sequences were clustered into 20 OTUs. The main genera were Sphingobacterium, Escherichia-Shigella, Alcaligenes, and Sediminibacter, Sphingobacterium replaced Pseudomonas as the genus with the highest abundance, and the proportion of Sphingobacterium reached $85.84 \%$. The result indicates that Sphingobacterium can tolerate higher concentrations of SMX than the other bacteria. As a result, the diversity in the enrichment gradually declined with the increase in SMX concentrations and passage generations. 


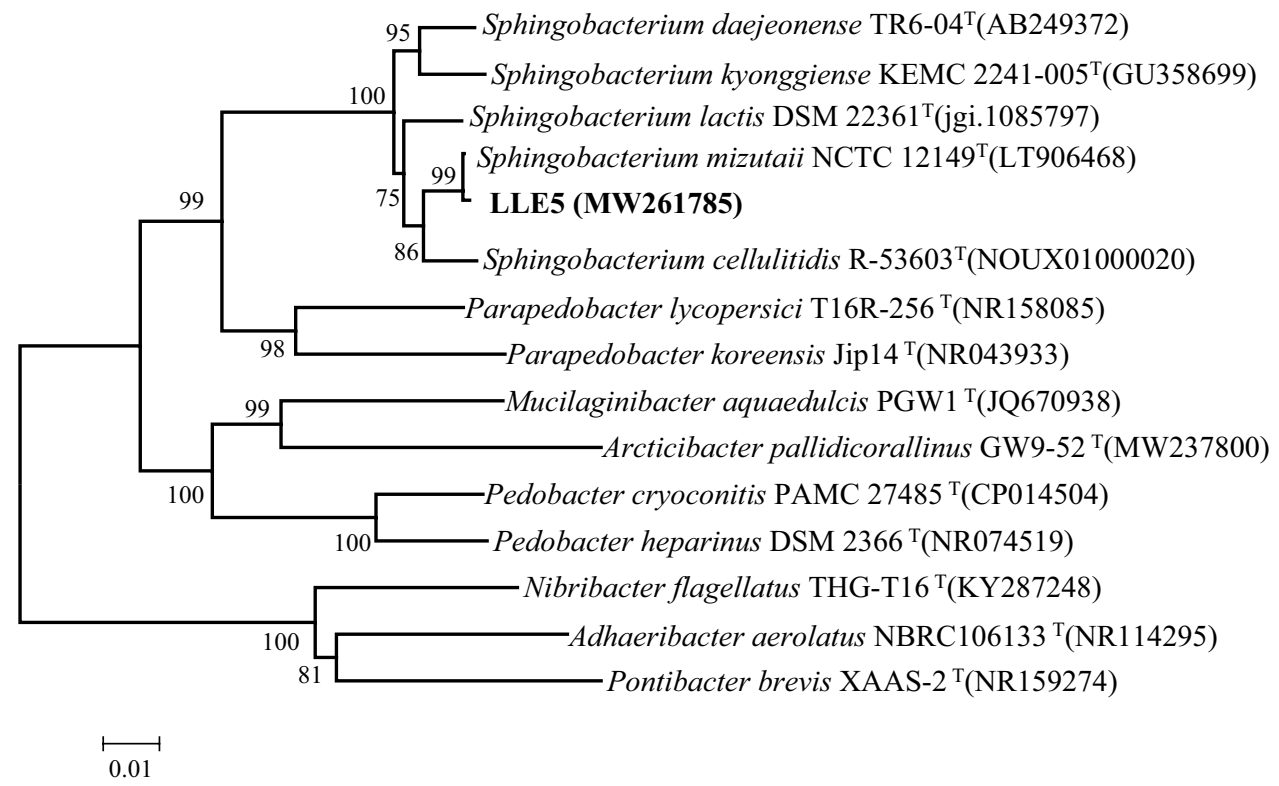

Figure 2. Neighbour-joining phylogenetic tree based on a comparison of the 16S rRNA gene sequences of S. mizutaii LLE5 and its closest relatives and several out-group strains. The numbers at the nodes indicate the percentages of bootstrap sampling derived from 1000 replications. GenBank accession numbers are given in parentheses. Bar, 0.01 nucleotide substitution per nucleotide position.

After the third and fourth passages, the 6003 sequences of SMX4 were clustered into 16 OTUs, and the main genera were Sphingobacterium, Alcaligenes, and Sediminibacter. The proportion of Sphingobacterium reached 93.68\% and became the dominant genera. This result provides strong evidence that Sphingobacterium contributed to SMX degradation. By contrast, the rapid reduction in the proportion of Pseudomonas and other bacteria indicates their lack of ability to tolerate high SMX concentrations.

Isolation and identification of SMX-degrading strains. Five strains that showed SMX degradation ability were isolated from SMX4 and were named LLE1-5. Among them, LLE5 utilized SMX as the sole carbon source in MSM and degraded $91.3 \%$ of $50 \mathrm{mg} / \mathrm{L}$ SMX in 7 days. It was selected for further morphological physiological, and biochemical analyses. LLE5 colonies on LB plate had smooth surfaces and clear edges and were light yellow in color. Under the microscope, cells of LLE5 featured short rods that were $0.6-1.8 \mu \mathrm{m}$ in length, $0.4-0.7 \mu \mathrm{m}$ in width, and were gram negative and nonmotile. LLE5 could utilize dextran, D-maltose, D-trehalose, D-fiber, gentian disaccharide, disaccharide, sucrose, D-pinobiose, and stachyose but not D-sorbitol, D-mannitol, and D-arabinol. The $16 \mathrm{~S}$ rRNA gene sequence of LLE5 was sequenced and submitted to GenBank with the accession number MW261785. Blast results showed that LLE5 belonged to the genus Sphingobacterium and clustered strongly with S. mizutaii NCTC $12149^{\mathrm{T}}$ (99.09\%, accession number LT906468) (Fig. 2). According to the results of morphological, physiobiochemical, and 16S rRNA evaluations, LLE5 was identified as a member of genus Sphingobacterium and named Sphingobacterium mizutaii. This is the first report that a Sphingobacterium species can degrade SMX. S. mizutaii LLE5 has been deposited in Guangdong Microbial Culture Collection of Center under number CGMCC 61038.

Optimization of the degradation conditions for LLE5. The influence of various environmental factors on the biodegradation efficiency was determined. S. mizutaii LLE5 can degrade SMX from 15 to $40{ }^{\circ} \mathrm{C}$ (Fig. 3a), with an optimum temperature of $30^{\circ} \mathrm{C}$. Although the temperature decreased to $15^{\circ} \mathrm{C}$, the degradation efficiency was more than $42.32 \%$. When the temperature rose to $40{ }^{\circ} \mathrm{C}$, the degradation efficiency was more than $58.65 \%$, and these results showed that $S$. mizutaii LLE5 has an obvious temperature adaptability. $\mathrm{pH}$ is another key factor affecting the degradation efficiency of the strain. The results of different $\mathrm{pH}$ on the degradation of the strain (Fig. 3b) show that LLE5 can degrade SMX in the $\mathrm{pH}$ range 4.0-9.0. At the optimal $\mathrm{pH}$ of 7.0, the degradation of SMX was $91.77 \%$. In addition, the degradation efficiency of LLE5 was more than $50 \%$ in the $\mathrm{pH}$ range 5.0-8.0, which indicated that the degradation efficiency of strain LLE5 was higher under neutral conditions. When the $\mathrm{pH}$ was reduced to 4.0 , the degradation efficiency was still $48.64 \%$, indicating that the strain was tolerant to acid conditions. When the $\mathrm{pH}$ was 9.0 , the degradation efficiency was $38.70 \%$, which indicated that LLE5 could be applied under neutral and acid conditions. The different initial inoculum amount on the degradation efficiency was analyzed (Fig. 3c). When the inoculation amount was $5 \times 10^{7} \mathrm{cfu} / \mathrm{mL}$, the degradation efficiency of S. mizutaii LLE5 was the highest, which was $92.89 \%$. However, when the initial inoculation amount was $2.0 \times 10^{7}-8.0 \times 10^{7} \mathrm{cfu} / \mathrm{mL}$, the degradation efficiency was more than $90 \%$, indicating that the degradation efficiency of $S$. mizutaii LLE5 did not change significantly with the change in inoculum amount. The initial concentration of SMX also influenced the degradation efficiency of S. mizutaii LLE5 (Fig. 3d). The degrada- 
a
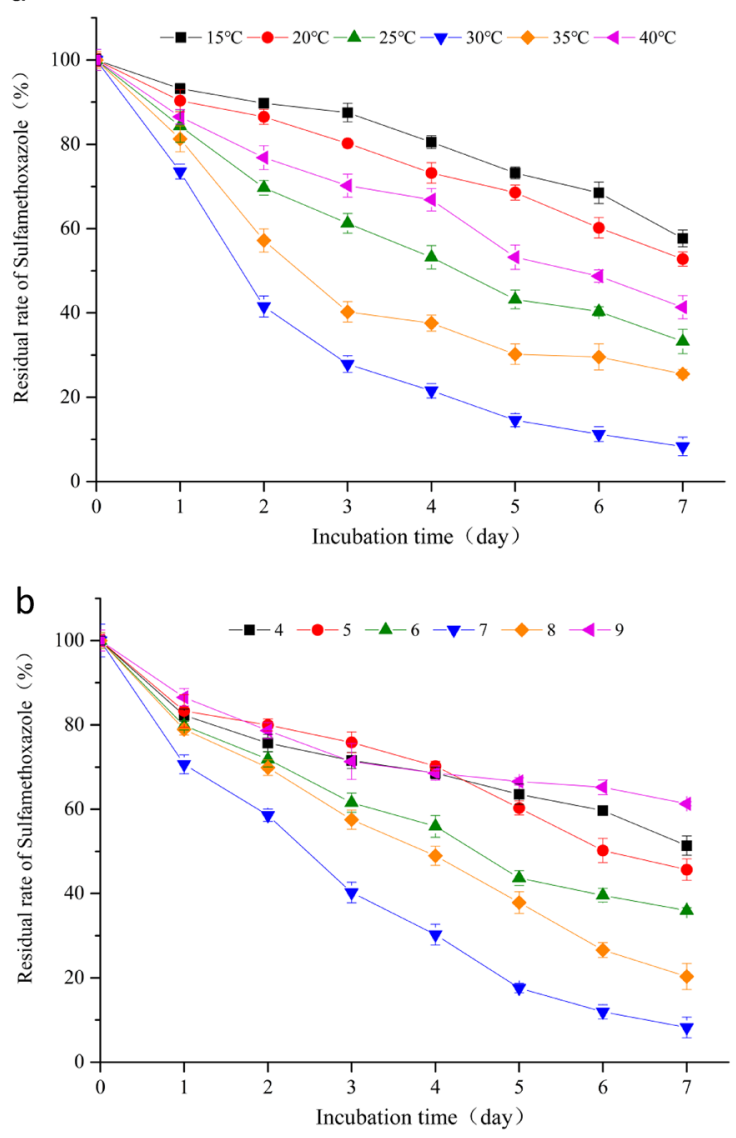

C

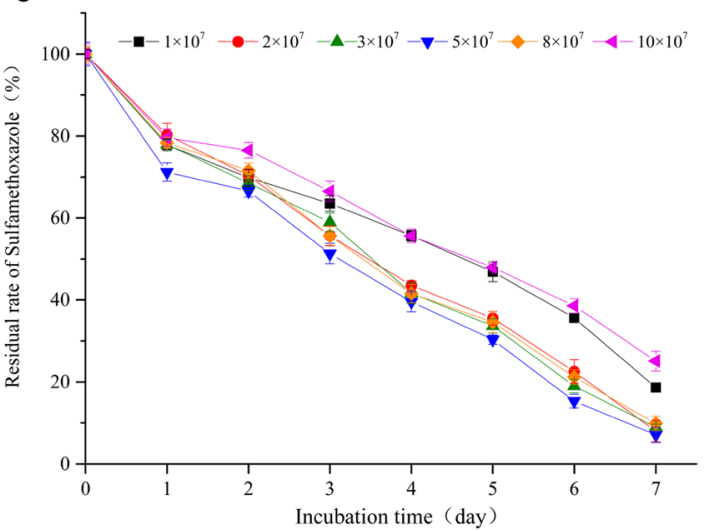

d

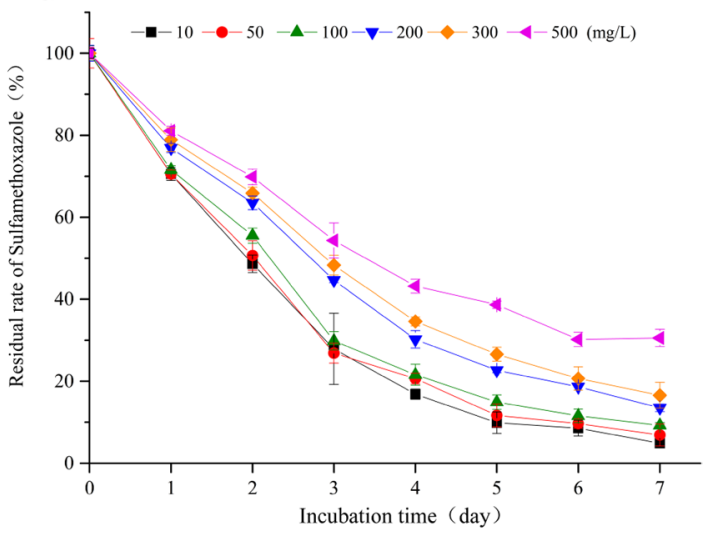

Figure 3. Degradation kinetics of SMX under different conditions (a) temperature $\left({ }^{\circ} \mathrm{C}\right),(\mathbf{b}) \mathrm{pH}$ level, (c) inoculum amount $\left(\times 10^{7} \mathrm{cfu} / \mathrm{mL}\right)$, and $(\mathbf{d})$ initial concentration $(\mathrm{mg} / \mathrm{L})$ of SMX. The symbols represent averages of triplicate experiments and the error bars indicate their corresponding standard deviations.

tion efficiency of S. mizutaii LLE5 with $10 \mathrm{mg} / \mathrm{L}$ SMX reached $95.14 \%$ and decreased to $83.42 \%$ when the SMX concentration reached $300 \mathrm{mg} / \mathrm{L}$. These results indicate that high concentrations of SMX inhibit the growth of S. mizutaii LLE5.

The temperature, $\mathrm{pH}$ levels and inoculation size were further designed through the response surface method in accordance with the results of single-factor experiments, and 17 degradation tests were carried out (Table 1). By the statistical analysis of data, the following second-degree polynomial equation was obtained to explain SMX biodegradation by $S$. mizutaii LLE5:

$$
Y_{1}=92.74-3.09 A-2.06 B+0.32 C+3.10 A B-1.07 A C-1.08 B C-9.40 A^{2}-8.40 B^{2}-0.12 C^{2}
$$

where $\mathrm{Y}_{1}$ represents the SMX degradation efficiency, and $\mathrm{A}, \mathrm{B}$, and $\mathrm{C}$ are the coded values for temperature, $\mathrm{pH}$ level, and inoculum amount respectively. Analysis of variance (ANOVA) for the fitted quadratic polynomial model is shown in Table 2. The model was signifcant $(P<0.05)$ with $R^{2}=0.9831$ and $\operatorname{Adj} R^{2}=0.9613$. The results of regression analysis indicated that $\mathrm{A}, \mathrm{B}, \mathrm{AB}, \mathrm{A}^{2}$ and $\mathrm{B}^{2}$ are significant model terms, whereas the $\mathrm{C}, \mathrm{AC}, \mathrm{BC}, \mathrm{C}^{2}$ are nonsignificant model terms. The same was confirmed from the Pareto chart (Fig. 4) in which higher effects were presented in the upper portion and then progress down to the lower effects. It directly shows that the most import factors influencing biodegradation efficiency were $\mathrm{A}, \mathrm{B}, \mathrm{AB}, \mathrm{A}^{2}$ and $\mathrm{B}^{2}$. The three-dimensional response surface was plotted to directly display the effects of the temperature and $\mathrm{pH}$ level on SMX biodegradation. At the theoretical maximum point of response surface (Fig. 5), the optimum conditions for SMX degradation by $S$. mizutaii LLE5 were $30.8^{\circ} \mathrm{C}$, pH 7.2, and inoculum amount of $3.5 \times 10^{7} \mathrm{cfu} / \mathrm{mL}$.

Degradation of SMX by strain LLE5. The degradation characteristics of SMX by S. mizutaii LLE5 under the optimal conditions were studied. The results indicated that the most efficient degradation was obtained during the first 3 days. On the third day, the degradation efficiency was $84.02 \%$, and the cell concentration was $68.95 \% \times 10^{7} \mathrm{cfu} / \mathrm{mL}$ (Fig. 6). The degradation efficiency was positively correlated with the cell growth density. At 5-7 days, the degradation efficiency of SMX gradually decreased and was accompanied by no further increase in S. mizutaii LLE5 cell density. Finally, the degradation efficiency of SMX with initial concentration of $50 \mathrm{mg} / \mathrm{L}$ was $93.87 \%$ after 7 days. This is the first report that Sphingobacterium mizutaii has a good degradation effect on SMX. S. mizutaii LLE5 can also degrade other sulfonamides, and the degradation efficiencies of strain LLE5 for sulfadiazine, sulfaguanidine, sulfamisoxazole, and sulfadimidine were 59.85\%, 51.68\%, 46.95\%, and 37.42\%, 


\begin{tabular}{|l|l|l|l|l|}
\hline Run & $\mathbf{X}_{\mathbf{1}}$ & $\mathbf{X}_{\mathbf{2}}$ & $\mathbf{X}_{\mathbf{3}}$ & Degradation efficiency (\%) \\
\hline 1 & 25 & 8 & 3 & 72.3 \\
\hline 2 & 25 & 5 & 3 & 84.8 \\
\hline 3 & 30 & 6.5 & 3 & 93.11 \\
\hline 4 & 25 & 6.5 & 1 & 84.5 \\
\hline 5 & 25 & 6.5 & 5 & 87.1 \\
\hline 6 & 30 & 8 & 5 & 82.6 \\
\hline 7 & 30 & 6.5 & 3 & 92.84 \\
\hline 8 & 35 & 5 & 3 & 71.4 \\
\hline 9 & 35 & 6.5 & 1 & 81.5 \\
\hline 10 & 30 & 5 & 5 & 86.7 \\
\hline 11 & 30 & 8 & 1 & 83.9 \\
\hline 12 & 30 & 6.5 & 3 & 93.87 \\
\hline 13 & 35 & 8 & 3 & 71.3 \\
\hline 14 & 35 & 6.5 & 5 & 79.8 \\
\hline 15 & 30 & 6.5 & 3 & 92.52 \\
\hline 16 & 30 & 5 & 1 & 83.7 \\
\hline 17 & 30 & 6.5 & 3 & 91.37 \\
\hline & & & \\
\hline
\end{tabular}

Table 1. Box-Behnken experimental design with three independent variables. $\mathrm{X}_{1}$ : temperature $\left({ }^{\circ} \mathrm{C}\right), \mathrm{X}_{2}: \mathrm{pH}$ level, $\mathrm{X}_{3}$ : inoculation amount $\left(\times 10^{7} \mathrm{cfu} / \mathrm{mL}\right)$.

\begin{tabular}{|l|c|c|c|c|l|}
\hline Source & Sum of squares & DF & Mean square & F value & P value \\
\hline $\mathrm{X}_{1}$ & 76.26 & 1 & 76.26 & 35.61 & 0.0006 \\
\hline $\mathrm{X}_{2}$ & 34.03 & 1 & 34.03 & 15.89 & 0.0053 \\
\hline $\mathrm{X}_{3}$ & 0.85 & 1 & 0.85 & 0.39 & 0.5499 \\
\hline $\mathrm{X}_{1} \mathrm{X}_{2}$ & 38.44 & 1 & 38.44 & 17.95 & 0.0039 \\
\hline $\mathrm{X}_{1} \mathrm{X}_{3}$ & 4.62 & 1 & 4.62 & 2.16 & 0.1852 \\
\hline $\mathrm{X}_{2} \mathrm{X}_{3}$ & 4.62 & 1 & 4.62 & 2.16 & 0.1852 \\
\hline $\mathrm{X}_{1} \mathrm{X}_{1}$ & 371.73 & 1 & 371.73 & 173.57 & 0.0001 \\
\hline $\mathrm{X}_{2} \mathrm{X}_{2}$ & 296.81 & 1 & 296.81 & 138.59 & 0.0011 \\
\hline $\mathrm{X}_{3} \mathrm{X}_{3}$ & 0.062 & 1 & 0.062 & 0.029 & 0.8701 \\
\hline Model & 869.49 & 9 & 96.61 & 45.11 & 0.0002 \\
\hline Error & 3.35 & 4 & 0.84 & & \\
\hline Total & 884.48 & 16 & & & \\
\hline
\end{tabular}

Table 2. Analysis of variance (ANOVA) for the fitted quadratic polynomial model. $P$ Value $<0.05$ indicates the model terms are significant.

respectively (Fig. 7). To elucidate the degradation ability of $S$. mizutaii LLE-5 against various sulfonamides, the degradation constant $(k)$ and half-life $\left(t_{1 / 2}\right)$ were determined by using the first-order kinetic model. Table 3 presents the kinetics parameters calculated from the model. The coefficient of determination $R^{2}$ varied from 0.9572 to 0.9924 indicating that the degradation data reliably fitted with the first-order kinetic model. Degradation rate constants $(k)$ varied from 0.0620 to $0.4247 \mathrm{~d}^{-1}$ that characterized the degradation process of various sulfonamides by strain LLE-5. Theoretical half-life $\left(t_{1 / 2}\right)$ of SMX, sulfaguanidine, sulfamisoxazole, and sulfadimidine was noted as $1.63,5.22,6.80,7.96$, and 11.18 days, respectively. These results show that $S$. mizutaii LLE5 has a broad specificity for the degradation of sulfonamides and has considerable potential for processing sulfonamide pollution in the environment.

Metabolic pathways of SMX degradation by S. mizutaii LLE5. The metabolites of SMX degraded by $S$. mizutaii LLE5 in MSM liquid medium were detected by HPLC/MS. According to the chemical structure of SMX and the mass spectrum, four candidate products were identified. These products were sulfanilamide $(171 \mathrm{~m} / \mathrm{z}), 4$-aminothiophenol $(124 \mathrm{~m} / \mathrm{z}), 5$-amino-3-methylisoxazole $(99 \mathrm{~m} / \mathrm{z})$, and aniline $(92 \mathrm{~m} / \mathrm{z})$. None of these products were detected when the culture medium only contained SMX and without $S$. mizutaii LLE5, indicating that they are SMX biodegradation metabolites. A possible metabolic pathway for SMX biodegradation by LLE5 was proposed (Fig. 8). SMX is first transformed into sulfanilamide and 5-amino-3-methylisoxazole through hydrogenation. Then, sulfanilamide is degraded via desulfurization into aniline and via deamination into 4-aminothiophenol. Although the ring-opening products of hydroquinone were not detected, it is still the first report of the pathway of SMX degradation by a Sphingobacterium strain. The candidate products were iden- 


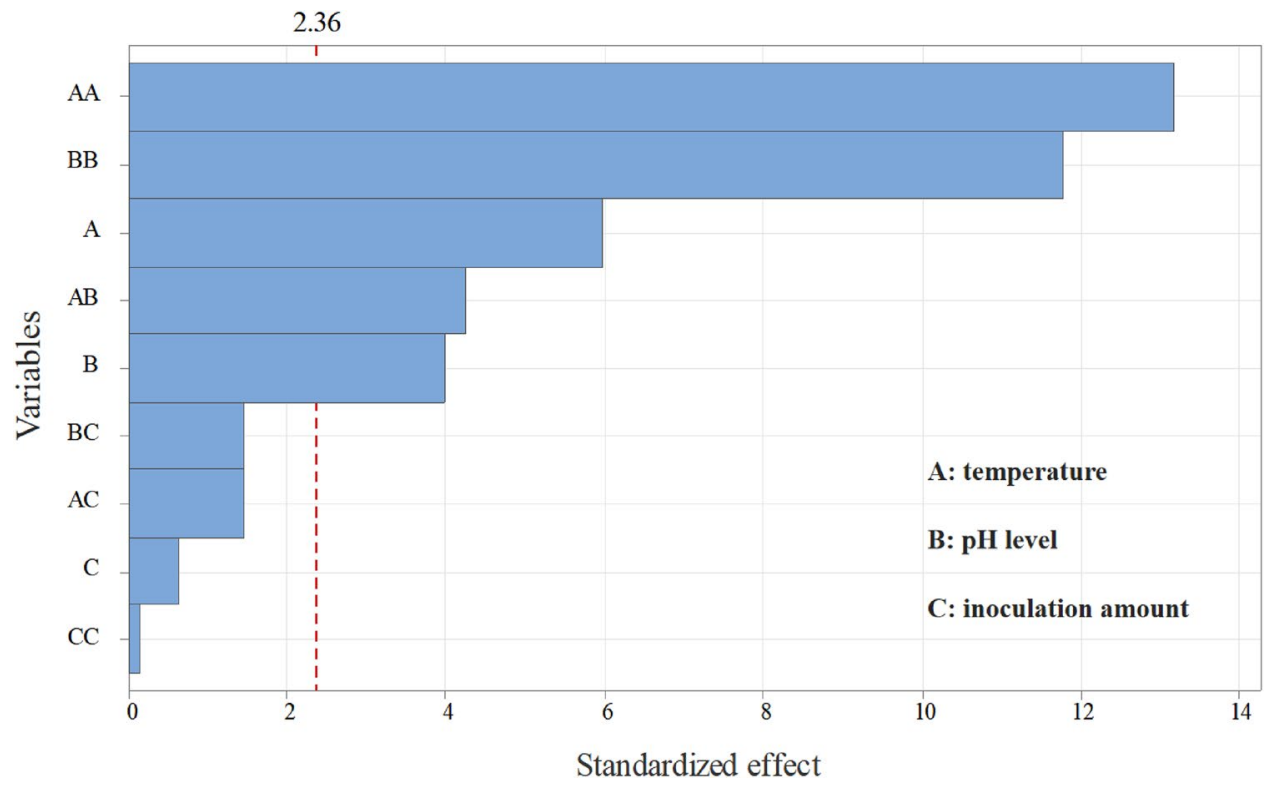

Figure 4. Pareto chart showing the effect of single factor (variables) biodegradation efficiency.

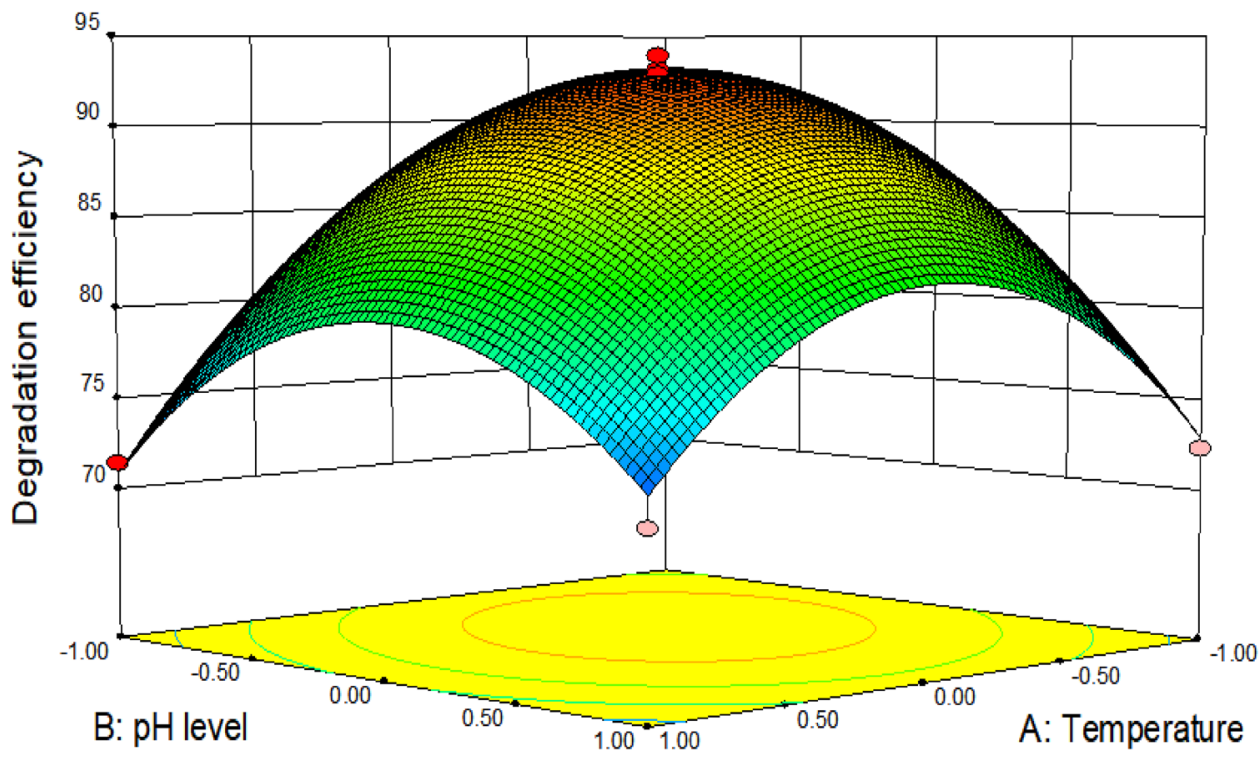

Figure 5. Response surface curves demonstrating the effects of medium temperature $\left({ }^{\circ} \mathrm{C}\right)$ and $\mathrm{pH}$ level on SMX biodegradation efficiency (\%) with an inoculum amount of S. mizutaii LLE5 at $3.5 \times 10^{7} \mathrm{cfu} / \mathrm{mL}$.

tified according to their $\mathrm{m} / \mathrm{z}$ values, SMX chemical properties, and existing reports. Standard samples of four intermediate products were also purchased to conduct parallel experiments.

\section{Discussion}

Sphingobacterium strains widely exist in natural environment ${ }^{20}$. Sphingobacterium is a kind of gram-negative bacteria and does not produce spores. Since the genus Sphingobacterium was proposed originally by Yabuuchi et al. in $1983^{21}$, new species have been discovered from a variety of environments, such as soil, plants, animals, and even clinical samples of ventricular fluid and urine. Among them, Sphingobacterum thalpohilum and Sphingobacterum multivarum can degrade petroleum hydrocarbons. For example, S. multivorum SWH-2 has a strong ability to degrade petroleum ${ }^{22}$. After ensuring optimal conditions, the normal growth and enzyme secretion and activity of $S$. multivorum SWH-2 will also change, which can further improve the oil degradation of the strain. In addition, most Sphingobacterium found thus far are resistant to a variety of antibiotics from microorganisms. However, there is no report on the direct degradation of antibiotics by Sphingobacterium. Sphingobacterium 


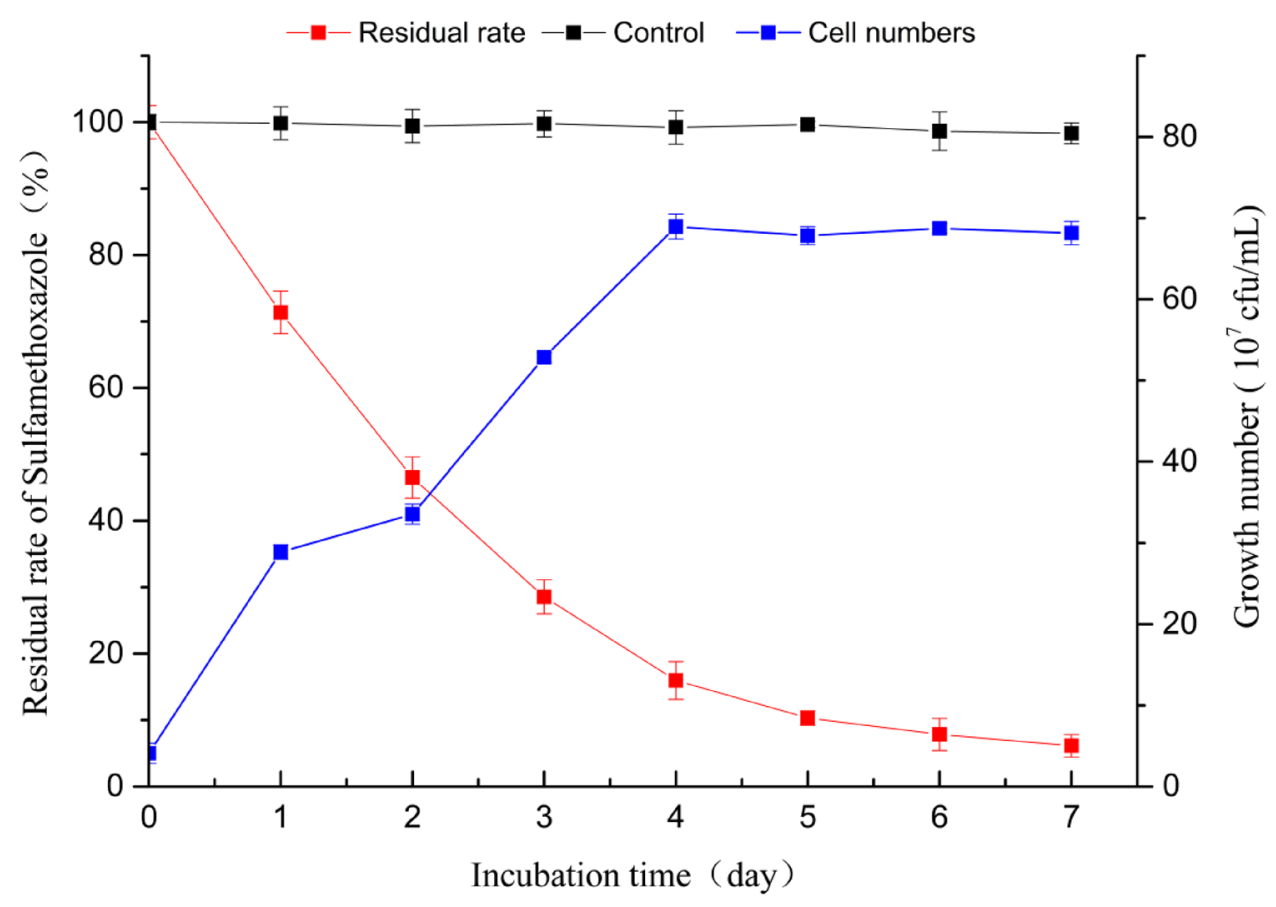

Figure 6. Growth of S. mizutaii LLE5 along time and degradation efficiency of SMX. The symbols represent averages of triplicate experiments and the error bars indicate their corresponding standard deviations.

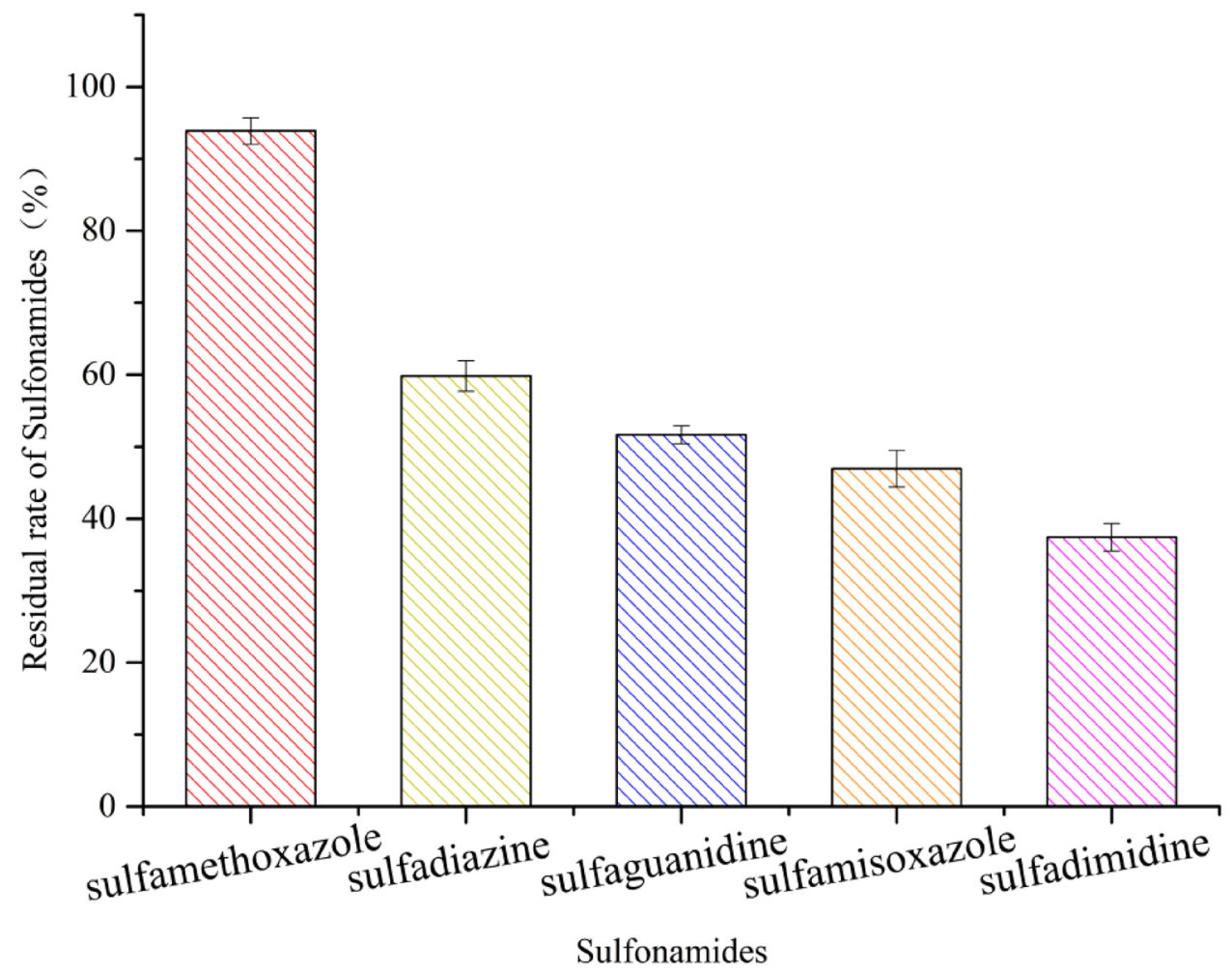

Figure 7. Degradation kinetics of various sulfonamides by S. mizutaii LLE5. The symbols represent averages of triplicate experiments and the error bars indicate their corresponding standard deviations. 


\begin{tabular}{|l|l|l|l|l|}
\hline Sulfonamides & Regression equation & $\mathbf{k}\left(\mathbf{d}^{-1}\right)$ & $\mathbf{t}_{\mathbf{1} / 2}(\mathbf{d})$ & $\boldsymbol{R}^{\mathbf{2}}$ \\
\hline Sulfamethoxazole & $C_{t}=51.3514 \times \mathrm{e}^{-0.4554 t}$ & 0.4247 & 1.63 & 0.9887 \\
\hline Sulfadiazine & $C_{t}=50.6351 \times \mathrm{e}^{-0.1327 \mathrm{t}}$ & 0.1327 & 5.22 & 0.9924 \\
\hline Sulfaguanidine & $C_{t}=50.2014 \times \mathrm{e}^{-0.1019 t}$ & 0.1019 & 6.80 & 0.9805 \\
\hline Sulfamisoxazole & $C_{t}=49.8941 \times \mathrm{e}^{-0.0871 t}$ & 0.0871 & 7.96 & 0.9572 \\
\hline Sulfadimidine & $C_{t}=50.1347 \times \mathrm{e}^{-0.0620 t}$ & 0.0620 & 11.18 & 0.9716 \\
\hline
\end{tabular}

Table 3. Kinetic parameters of various sulfonamides degradation by $S$. mizutaii LLE- 5 . k represents degradation constant $\left(\mathrm{d}^{-1}\right) ; \mathrm{t}_{1 / 2}$ represents half-time $(\mathrm{d}) ; R^{2}$ represents correlation coeffificient; $\mathrm{Ct}$ is the concentration $(\mathrm{mg} / \mathrm{L})$ of sulfonamides at time $t$.

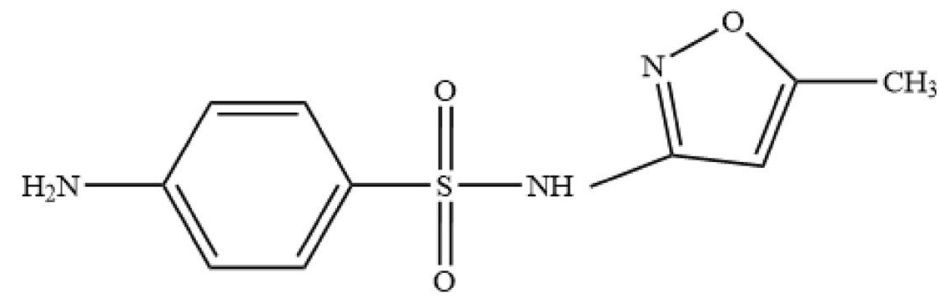

4-Amino- $N$-(5-methylisoxazol-3-yl)benzensulfonamide

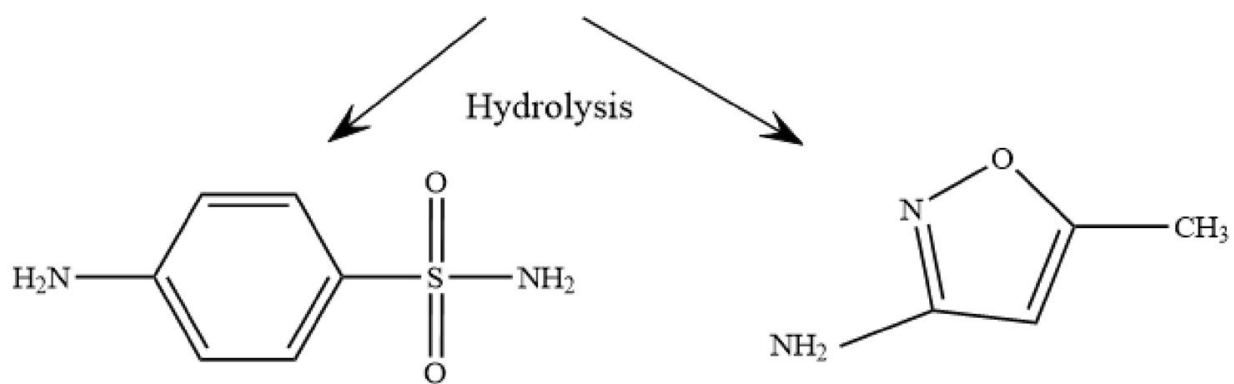

Sulfanilamide

5-Amino-3-methylisoxazole
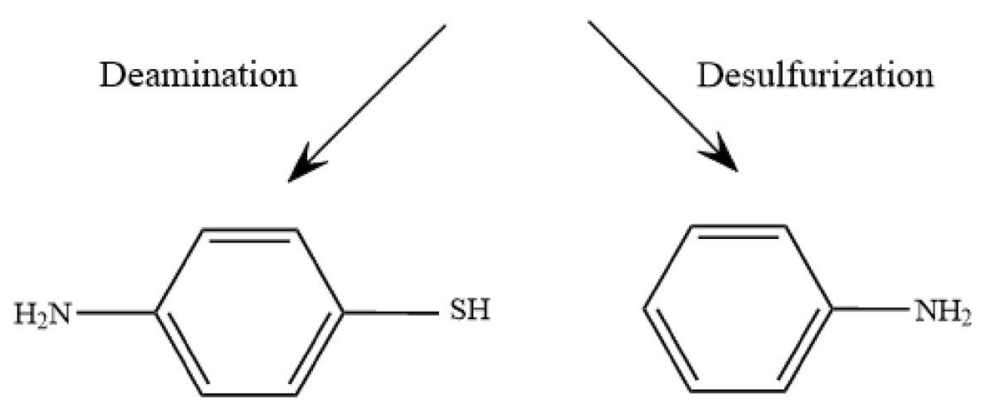

\section{4-Aminobenzesulfonamide}

Aniline

Figure 8. Proposed pathway for the degradation of SMX by S. mizutaii LLE5.

mizutaii isolated in this study is the first Sphingobacterium reported to degrade SMX. The discovery of this bacterium not only expanded the functional range of Sphingobacterium but also provided valuable microbial resources for the remediation of SMX contaminated aquaculture environment.

In recent years, a number of strains with good SMX degradation ability, such as Planococcus kocurii O516, Achromobacter sp. JL9, Phanerochaete chrysosporium, and Acinetobacter sp., have been isolated from soil, activated sludge, and aquaculture water. Compared with the reported strain, the strain LLE-5 had a better environmental range and tolerance. The study on the influence of environmental factors on the degradation effect indicates that strain LLE- 5 can degrade SMX at the temperature range $15-40{ }^{\circ} \mathrm{C}$ and at $\mathrm{pH} 4-9$. This finding provides a basis for the practical application of the strain in the future. The physiological and biochemical results 
showed that LLE-5 could use a variety of carbon sources. The bacteria have strong adaptability to the environment, rich types of nutrition metabolism, and potential to degrade other complex compounds.

The existing reports show that most strains of Sphingobacterium cannot produce antibiotics but are resistant to antibiotics, which is an important factor for the Sphingobacterium to adapt to extreme environments ${ }^{23}$. Antibiotics are compounds that inhibit the growth of microorganisms. Thus, degrading bacteria need to have antibiotic resistance. Some studies have shown that Klebsiella pneumoniae can use chloramphenicol as the sole carbon source for growth ${ }^{24}$. However, the drug sensitivity test showed that these strains were sensitive to chloramphenicol, which indicated that the drug resistance and drug degradation processes were two independent pathways. Drug-resistant bacteria can only resist antibiotics and avoid growth inhibition. By contrast, antibiotic-degrading bacteria resist antibiotics and produce degrading enzymes such as monooxygenase, esterase, or hydrolase to degrade the drug. We sequenced the whole genome of strain LLE5 and found some genes that might be involved in SMX degradation (data not shown). Their functions will be identified in our future work. According to the degradation products of SMX by S. mizutaii LLE-5, the strain mainly transformed SMX into sulfanilamide and 5-amino-3-methylisoxazole through hydrogenation. Then, sulfanilamide is degraded via desulfurization into aniline and via deamination into 4-aminothiophenol. The results showed that the strain had a clear degradation function of sulfamethoxazole and had a good tolerance. However, SMX could not be completely mineralized in terms of degradation products. This work is the first to report the pathway of SMX degradation by a Sphingobacterium strain and provided a certain reference for the study of microbial degradation of SMX.

\section{Methods}

Chemicals and media. SMX (analytical standard, $>99.5 \%$ ), HPLC-grade methanol, and acetonitrile were purchased from Sinopharm Chemical Reagent Beijing Co., Ltd. All other reagents used in the present study were of analytical grade. Mineral salt medium (MSM) and Luria-Bertani (LB) medium were used as described by Song et $\mathrm{al}^{25}$. Agar plates were prepared by adding $1.5 \%(\mathrm{w} / \mathrm{v})$ agar into the liquid media.

Enrichment culture and high-throughput sequencing. Activated sludge samples were collected from the wastewater treatment pond of a pharmaceutical factory in Liaoyang City, Liaoning Province, China. $10.0 \mathrm{~g}$ of sample was suspended in $100 \mathrm{~mL}$ of MSM supplemented with $100 \mathrm{mg} / \mathrm{L}$ SMX. Then, the sample was incubated on a rotary incubator shaker at $30^{\circ} \mathrm{C}$ and $100 \mathrm{r} / \mathrm{min}$ in the dark. After 7 days, $10 \mathrm{~mL}$ of the sample was transferred into fresh MSM supplemented with $200 \mathrm{mg} / \mathrm{L}$ of SMX and incubated for another 7 days. After repeating this process four times, the SMX concentration in the fourth generation increased to $500 \mathrm{mg} / \mathrm{L}$. The activated sludge sample was designated as SMXY, and the four generations of SMX enrichment cultures were designated as SMX1, SMX2, SMX3, and SMX4. The total DNA of SMXY and SMX1-4 were extracted using a FastDNA SPIN kit for soil (MP, Biomedicals, USA). After purification, the 16S rRNA distinct regions V3-V4 were amplified with the following primers: 338F (5'-ACTCCTACGGGAGGCAGCA-3') and 806R (5'-GGACTACHVGGG TWTCTAAT-3'). The polymerase chain reaction (PCR) system was designed as described by Li et $\mathrm{al}^{26}$. The PCR product was sent to Biomark Gene Technology Company for library construction and high-throughput sequencing. The obtained sequence data were analyzed using Arch software (http://www.drive5.com/usearch/). The identity of sequences $\geq 97 \%$ was assigned to an operational taxonomic unit (OTU), and each OTU was considered to represent a species. The relative abundance of OTUs in the samples was statistically analyzed using R software (https://www.R-project.org) ${ }^{27}$.

Isolation and identification of SMX-degrading bacteria. According to the results of high-throughput sequencing, the fourth-generation enrichment sample SMX4 containing the most Sphingobacterium cells was used for isolation of SMX-degrading bacteria. Then, $100 \mu \mathrm{L}$ of SMX4 was spread on the MSM solid plate containing $100 \mathrm{mg} / \mathrm{L} \mathrm{SMX}$ and incubated at $30^{\circ} \mathrm{C}$. The rapidly growing colonies on the plate with different morphologies were selected and restreaked three times to obtain pure cultures. The degradation efficiency of the isolates was determined by a high-performance liquid chromatography (HPLC) system (1260, Agilent, Santa Clara, CA) equipped with an eclipse Plus $\mathrm{C}_{18}$ column. $10 \mathrm{~mL}$ of the culture and equal volume of ethyl acetate were added into a $50 \mathrm{~mL}$ centrifuge tube and mixed using a vortex mixer for $1 \mathrm{~min}$. Then, the centrifuge tube was placed in a shaker, mixed at $220 \mathrm{r} / \mathrm{min}$ for $30 \mathrm{~min}$, and centrifuged at $5000 \mathrm{~g}$ for $8 \mathrm{~min}$. The upper phase was filtered with a $0.22 \mu \mathrm{m}$ membrane for HPLC analysis. The elution comprised a mixture of methanol, formic acid $(85 / 15 / 0.1, \mathrm{v} / \mathrm{v} / \mathrm{v})$, and distilled water, running at the flow rate of $1.0 \mathrm{~mL} / \mathrm{min}$. The injection volume was $10 \mu \mathrm{L}$, and the column temperature was $30^{\circ} \mathrm{C}^{28}$. The isolate with the highest degradation efficiency was selected for further analysis. Morphology was investigated using a light microscope (BX-51; Olympus, Japan). The carbohydrate assimilation of isolate was conducted with Biolog GEN3 plates following the analytical methods described by Hobbie et $\mathrm{al}^{29}$. The genomic DNA of the isolate was extracted and was used as a template for 16S rDNA amplification as described by Ruan et $\mathrm{al}^{30}$. The universal primers were 27F ( $5^{\prime}$-AGAGTTTGATCCTGGCTCAG-3') and 1492R (5'ACGGHTACCTTGTTTACGACTT-3'). The purified PCR product was sequenced by Bomad Technology (Beijing, China). The obtained sequence was deposited in GenBank. The strain was further identified by performing multiple sequence alignment using Clustal X sofware, and phylogenetic relationships were analyzed via the neighbor-joining (NJ) method with MEGA 6 software ${ }^{31}$.

Inoculum preparation. Before the degradation experiment, strain activation was performed. S. mizutaii LLE-5 was inoculated into $100 \mathrm{~mL}$ of LB medium and incubated at $30^{\circ} \mathrm{C}$ on a rotary shaker at $150 \mathrm{rpm}$. After $12 \mathrm{~h}$, the bacterial cells were harvested by centrifugation at $4000 \mathrm{~g}$ for $10 \mathrm{~min}$. The precipitate was washed two times by phosphate buffered saline (PBS) solution and suspended for subsequent studies ${ }^{32}$. 
Optimization of the SMX-degrading conditions. The effect of single environmental factors on the biodegradation efficiency was evaluated by analyzing the following parameters and their ranges ${ }^{33}$ : temperature $\left(15^{\circ} \mathrm{C}, 20^{\circ} \mathrm{C}, 25^{\circ} \mathrm{C}, 30^{\circ} \mathrm{C}, 35^{\circ} \mathrm{C}\right.$, and $\left.40{ }^{\circ} \mathrm{C}\right)$; initial medium $\mathrm{pH}(4,5,6,7,8$, and 9$)$; inoculum amount $\left(1.0 \times 10^{7}\right.$, $2.0 \times 10^{7}, 3.0 \times 10^{7}, 5.0 \times 10^{7}, 8.0 \times 10^{7}$, and $\left.10.0 \times 10^{7} \mathrm{cfu} / \mathrm{mL}\right)$; and initial concentration $(10,50,100,200,300$ and $500 \mathrm{mg} / \mathrm{L}$ ). The sterile distilled water was supplemented every $12 \mathrm{~h}$ according to the loss of weight. Selected single environmental factors and their interactions were further optimized via response surface methodology (RSM) analysis based on Box - Behnken design ${ }^{34}$. The second order polynomial equation is expressed as follows:

$$
Y_{i}=b_{o}+\sum b_{i} X_{i}+\sum b_{i j} X_{i} X_{j}+\sum b_{i i} X_{i}^{2}
$$

where $Y_{i}$ refers to the predicted response, $X_{i}$ and $X_{j}$ are variables, $b_{o}$ is a constant, $b_{i}$ denotes the linear coefficient, $b_{i i}$ represents the quadratic coefficient, and $b_{i j}$ corresponds to the interaction coefficient.

Biodegradation tests. The SMX degradation test was performed under optimal conditions. S. mizutaii LLE-5 cells were inoculated into $100 \mathrm{~mL}$ of MSM supplemented with $50 \mathrm{mg} / \mathrm{L}$ of SMX. The residual of SMX and cell numbers of $S$. mizutaii LLE-5 were detected every day. The control was inoculated with killed cells of $S$. mizutaii LLE-5. Each test was conducted in triplicate ${ }^{35}$. The ability of $S$. mizutaii LLE- 5 to degrade other structurally similar sulfonamides, including sulfadiazine, sulfaguanidine, sulfamisoxazole, and sulfadimidine, were evaluated. Analytical methods were the same as above described. The first-order kinetic model (Eq. 3) was created to elucidate sulfonamides degradation efficiency of $S$. mizutaii LLE- $5^{36}$.

$$
C_{t}=C_{0} \times \mathrm{e}^{-\mathrm{kt}}
$$

where $C_{0}$ is the initial concentration of sulfonamides at time zero, $C_{t}$ is the concentration of sulfonamides at time $t, k$ is the degradation rate constant $\left(\mathrm{d}^{-1}\right)$.

The theoretical half-life $\left(t_{1 / 2}\right)$ values of different sulfonamides were calculated by Eq. (4).

$$
t_{1 / 2}=\frac{\ln (2)}{k}
$$

where $\ln 2$ is the natural logarithm of 2 and $k$ is degradation rate constant $\left(\mathrm{d}^{-1}\right)$.

Identification of SMX biodegradation intermediates. Intermediates generated during SMX degradation by $S$. mizutaii LLE-5 in MSM were analyzed by HPLC-MS (AB Sciex QTRAP 5500, USA). The extraction method was same as described above. The flow rate was $0.2 \mathrm{~mL} / \mathrm{min}$, the mobile phase A was $0.1 \%$ formic acid $(\mathrm{V} / \mathrm{V})$, and $\mathrm{B}$ was methanol. The targeted screening gradient elution program was: $0-2 \mathrm{~min}, 95 \% \mathrm{~A} ; 2-25 \mathrm{~min}$, 95\%-5\% A; 25-35 min, 5\% A; and 35-40 min, 95\% A. The sample injection volume was $20 \mu \mathrm{L}$, and the column temperature was $30^{\circ} \mathrm{C}$. The mass spectrometry conditions were: DuoSprayTM ion source, electrospray ionization (ESI), positive ion mode scanning, the ion source temperature was $550{ }^{\circ} \mathrm{C}$, the spray voltage was $5500 \mathrm{~V}$, and curtain air (CUR) was $35 \mathrm{psi}$. The accumulation time was $0.25 \mathrm{~s}$, and the collision voltage was $(35 \pm 15) \mathrm{eV}$.

Received: 3 August 2021; Accepted: 6 October 2021

Published online: 30 November 2021

\section{References}

1. Han, Q. F. et al. Distribution, combined pollution and risk assessment of antibiotics in typical marine aquaculture farms surrounding the Yellow Sea, North China. Environ. Int. 138, 105551 (2020).

2. Zhang, L. et al. Biodegradation mechanisms of sulfonamides by Phanerochaete chrysosporium: Luffa fiber system revealed at the transcriptome level. Chemosphere 266, 129194 (2021).

3. Shah, S. et al. Recent advances in medicinal chemistry of sulphonamides: Rational design as anti-tumoral, anti-bacterial and antiinflammatory agents. Mini. Rev. Med. Chem. 13, 70-86 (2013).

4. Cribb, A. \& Spielberg, S. Sulfamethoxazole is metabolized to the hydroxylamine in humans. Clin. Pharmacol. Ther. 51, 522-526 (1992).

5. Mulla, S. I. et al. Biodegradation of sulfamethoxazole in bacteria from three different origins. J. Environ. Manage. 206, 93-102 (2018).

6. Su, T., Deng, H., Benskin, J. P. \& Radke, M. Biodegradation of sulfamethoxazole photo-transformation products in a water/sediment test. Chemosphere 148, 518-525 (2016).

7. Xiong, J. Q. et al. Combined effects of sulfamethazine and sulfamethoxazole on a freshwater microalga, Scenedesmus obliquus: Toxicity, biodegradation, and metabolic fate. J. Hazard Mater. 370, 138-146 (2019).

8. Li, T. et al. Enhanced degradation of sulfamethoxazole by a novel Fenton-like system with significantly reduced consumption of $\mathrm{H}_{2} \mathrm{O}_{2}$ activated by g- $\mathrm{C}_{3} \mathrm{~N}_{4} \mathrm{MgO}$ composite. Water Res. 190, 116777 (2021).

9. Goncalves, M. G. et al. Relationship of the physicochemical properties of novel $\mathrm{ZnO} /$ biochar composites to their efficiencies in the degradation of sulfamethoxazole and methyl orange. Sci. Total Environ. 748, 141381 (2021).

10. Hong, M., Wang, Y. \& Lu, G. UV-Fenton degradation of diclofenac, sulpiride, sulfamethoxazole and sulfisomidine: Degradation mechanisms, transformation products, toxicity evolution and effect of real water matrix. Chemosphere 258, 127351 (2020).

11. Carneiro, R. B. et al. Influence of organic loading rate on ciprofloxacin and sulfamethoxazole biodegradation in anaerobic fixed bed biofilm reactors. J. Environ. Manage. 273, 111170 (2020).

12. Rodrigues, D. et al. Biodegradation of sulfamethoxazole by microalgae-bacteria consortium in wastewater treatment plant effluents. Sci. Total Environ. 749, 141441 (2020).

13. Liang, D. H. \& Hu, Y. Simultaneous sulfamethoxazole biodegradation and nitrogen conversion by Achromobacter sp. JL9 using with different carbon and nitrogen sources. Bioresour. Technol. 293, 122061 (2019). 
14. Gao, N. et al. Simultaneous removal of ciprofloxacin, norfloxacin, sulfamethoxazole by co-producing oxidative enzymes system of Phanerochaete chrysosporium and Pycnoporus sanguineus. Chemosphere 195, 146-155 (2018).

15. Jia, Y. et al. Sulfamethoxazole degradation in anaerobic sulfate-reducing bacteria sludge system. Water Res. 119, 12-20 (2017).

16. Reis, P. J. et al. Biodegradation of sulfamethoxazole and other sulfonamides by Achromobacter denitrificans PR1. J. Hazard Mater. 280, 741-749 (2014)

17. Jiang, B. et al. Biodegradation and metabolic pathway of sulfamethoxazole by Pseudomonas psychrophila HA-4, a newly isolated cold-adapted sulfamethoxazole-degrading bacterium. Appl. Microbiol. Biotechnol. 98(10), 4671-4681 (2014).

18. Yang, F. et al. Effects of biochar on biodegradation of sulfamethoxazole and chloramphenicol by Pseudomonas stutzeri and Shewanella putrefaciens: Microbial growth, fatty acids, and the expression quantity of genes. J. Hazard Mater. 406, 124311 (2021).

19. Wang, S. \& Wang, J. Biodegradation and metabolic pathway of sulfamethoxazole by a novel strain Acinetobacter sp. Appl. Microbiol. Biotechnol. 102(1), 425-432 (2018).

20. Naka, T. et al. Structural analysis of sphingophospholipids derived from Sphingobacterium spiritivorum, the type species of genus Sphingobacterium. Biochim. Biophys. Acta. 1635(2-3), 83-92 (2003).

21. Wauters, G. et al. Isolates belonging to CDC group II-i belong predominantly to Sphingobacterium mizutaii Yabuuchi et al. 1983: Emended descriptions of S. mizutaii and of the genus Sphingobacterium. Int. J. Syst. Evol. Microbiol. 62(Pt 11), 2598-2601 (2012).

22. Abro, A. H. et al. Sphingobacterium multivorum bacteremia and acute meningitis in an immunocompetent adult patient: A case report. Iran Red Crescent. Med. J. 18(9), e387502016 (2016).

23. Barahona, F. \& Slim, J. Sphingobacterium multivorum: Case report and literature review. New Microbes New Infect. 7, 33-36 (2015).

24. Srinivasan, V. B., Vaidyanathan, V., Mondal, A. \& Rajamohan, G. Role of the two component signal transduction system CpxAR in conferring cefepime and chloramphenicol resistance in Klebsiella pneumoniae NTUH-K2044. PLoS ONE 7(4), e33777 (2012).

25. Song, J. et al. Pathway and kinetics of malachite green biodegradation by Pseudomonas veronii. Sci. Rep. 10(1), 4502 (2020).

26. Li, M. et al. Insight into the characteristics and new mechanism of nicosulfuron biodegradation by a Pseudomonas sp. LAM1902. J. Agric. Food Chem. 68(3), 826-837 (2020).

27. Zhou, S. et al. Nicosulfuron biodegradation by a novel cold-adapted strain Oceanisphaera psychrotolerans LAM-WHM-ZC. J. Agric. Food Chem. 65(47), 10243-10249 (2017).

28. Larcher, S. \& Yargeau, V. Biodegradation of sulfamethoxazole by individual and mixed bacteria. Appl. Microbiol. Biotechnol. 91(1), 211-218 (2011).

29. Hobbie, E. A. et al. Carbohydrate use and assimilation by litter and soil fungi assessed by carbon isotopes and BIOLOG assays. Soil Biol. Biochem. 35, 303-311 (2003).

30. Ruan, Z. et al. Isolation and characterization of a novel cinosulfuron degrading Kurthia sp. from a methanogenic microbial consortium. Bioresour. Technol. 147, 477-483 (2013).

31. Ruane, N. M. et al. Isolation of Streptococcus agalactiae and an aquatic birnavirus from doctor fish Garra rufa L. Ir. Vet. J. 66(1), 16 (2013).

32. He, Z. et al. Biodegradation of feather waste keratin by the keratin-degrading strain Bacillus subtilis 8. J. Microbiol. Biotechnol. 28(2), 314-322 (2018).

33. Wang, S., Zhang, C. \& Yan, Y. Biodegradation of methyl parathion and p-nitrophenol by a newly isolated Agrobacterium sp. strain Yw12. Biodegradation 23(1), 107-116 (2012).

34. Wang, W. et al. Optimization of reactions between reducing sugars and 1-phenyl-3-methyl-5-pyrazolone (PMP) by response surface methodology. Food Chem. 254, 158-164 (2018).

35. Bhatt, P., Huang, Y., Zhan, H. \& Chen, S. Insight into microbial applications for the biodegradation of pyrethroid insecticides. Front Microbiol. 10, 1778 (2019).

36. Cycon, M., Zmijowska, A. \& Piotrowska-Seget, Z. Enhancement of deltamethrin degradation by soil bioaugmentation with two different strains of Serratiamarcescens. Int. Environ. Sci. Technol. 11, 1305-1316 (2014).

\section{Acknowledgements}

This work was supported by Open Research Fund of Key Laboratory of Healthy Freshwater Aquaculture, Ministry of Agriculture and Rural Affairs (ZJK202101), Central Public-interest Scientific Institution Basal Research Fund, (CAFS, No. 2021 A003) and National Key Research and Development Program of China (No. 2017YFC1600704).

\section{Author contributions}

Y.M. and Z.R. conceived and designed research. J.S., G.H., L.L., H.Z., and D.Z. conducted experiments. J.X., X.L. and Z. Y. analyzed and discussed the data. J.S. wrote the manuscript. All authors read and approved the manuscript.

\section{Competing interests}

The authors declare no competing interests.

\section{Additional information}

Supplementary Information The online version contains supplementary material available at https://doi.org/ 10.1038/s41598-021-02404-x.

Correspondence and requests for materials should be addressed to Z.R. or Y.M.

Reprints and permissions information is available at www.nature.com/reprints.

Publisher's note Springer Nature remains neutral with regard to jurisdictional claims in published maps and institutional affiliations. 
(c) (i) Open Access This article is licensed under a Creative Commons Attribution 4.0 International cc) License, which permits use, sharing, adaptation, distribution and reproduction in any medium or format, as long as you give appropriate credit to the original author(s) and the source, provide a link to the Creative Commons licence, and indicate if changes were made. The images or other third party material in this article are included in the article's Creative Commons licence, unless indicated otherwise in a credit line to the material. If material is not included in the article's Creative Commons licence and your intended use is not permitted by statutory regulation or exceeds the permitted use, you will need to obtain permission directly from the copyright holder. To view a copy of this licence, visit http://creativecommons.org/licenses/by/4.0/.

(C) The Author(s) 2021, corrected publication 2021 